\title{
Astrophyisical Neutrino at Hyper-Kamiokande
}

\section{Takatomi Yano*i}

Department of Physics, Kobe University

1-1 Rokko-dai, Nada, Kobe, Hyogo, 657-8501 JAPAN E-mail:

tyanodphys.sci.kobe-u.ac.jp

\begin{abstract}
Hyper-Kamiokande (Hyper-K) is a proposed next generation underground large water Cherenkov detector. We propose to build two cylindrical water tanks in our experimental period, filled with ultra pure water and surrounded with newly developed photo sensors. In total, it will provide the fiducial volume of 0.19-0.37 Mt. The energies, positions, directions and types of charged particles produced by neutrino interactions are detected using its Cherenkov light in water. Our detector will be located at deep underground to reduce the cosmic muon flux and its spallation products, which is a dominant background for the analysis of the low energy astrophysical neutrinos.

With the fruitful physics research programs planned for the accelerator neutrinos, atomospheric neutrinos and nucleon decay, Hyper-K will play a considerable role in the next neutrino physics frontier, even in the neutrino astrophysics. It will provide remarkable information for both of particle physics and astrophysics with its large statistics of astrophysical neutrino measurements, i.e., solar neutrino, supernova burst neutrinos and supernova relic neutrino. Here, we will discuss about physics potential of Hyper-K at neutrino astrophysics and expected performance of the detector.
\end{abstract}

35th International Cosmic Ray Conference - ICRC2017

10-20 July, 2017

Bexco, Busan, Korea

\footnotetext{
${ }^{*}$ Speaker.

${ }^{\dagger}$ for the Hyper-Kamiokande Proto-Collaboration
} 


\section{Introduction}

Hyper-Kamiokande (Hyper-K, HK) is a next generation water Cherenkov detector planned in Japan [1, 2, 3], as a successor of the Super-Kamiokande (Super-K, SK) experiment [4]. With the dimensions of the $74 \mathrm{~m}(\mathrm{D}) \times 60 \mathrm{~m}(\mathrm{H})$ for each, two cylindrical water tanks provide the fiducial (total) volume of 0.19 (0.52) million metric tons per tank (Figure 1). They are 8 (5) times larger than that of Super-K. The target date for beginning the measurement with first tank is 2026. The measurement with the second tank will begin 6 years after that. An inner detectors are surrounded by 40,000 photodetectors with 20 -inch diameter. 6,700 photodetectors with 8 -inch diameter are also provided for an outer veto detector to reject cosmic-ray muons. The 20-inch photodetector, Box\&Line photo-multiplier tube Hamamatsu R12860, is newly developed for HK, to achieve twice larger detection efficiency for Cherenkov photons, the superior photon counting and timing resolution compared to that of SK (Hamamatsu R3600) [5]. It also has the high pressure tolerance for the usage below $60 \mathrm{~m}$ depth of water. The multiple-photosensor unit is also in our R\&D for the outer detector [5, 6]. The detector will be located underground at Kamioka mine in Gifu Prefecture, with an overburden of $\sim 650$ meters or more of rock, which is equivalent to 1,750 meters or more of water. Charged particles, such as the products of neutrino interactions, are detected with the emitted Cherenkov photons. The number of photons and their arrival times on the photodetectors are used to reconstruct the energy and the vertex of the particle, respectively. Hyper-K has various physics topics: search for $\mathrm{CP}$ violation in neutrinos, precise study of neutrino oscillations including determination of mass hierarchy and $\theta_{23}$ octant with beam and atmospheric neutrinos, search for nucleon decay and observations of astrophysical neutrinos.

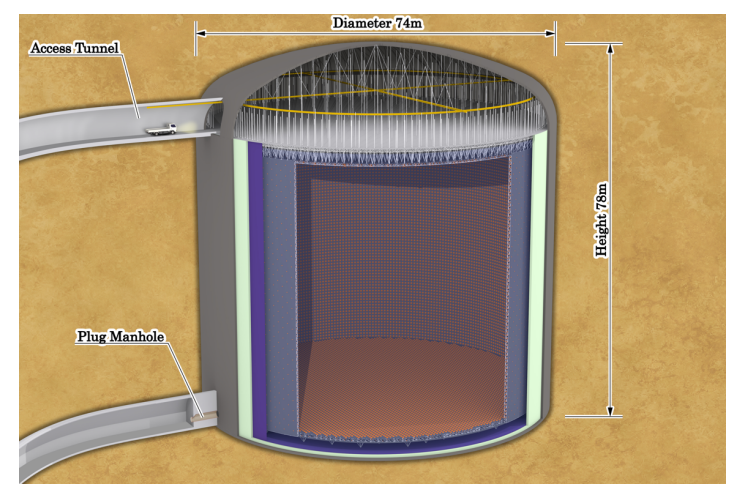

Figure 1: Schematic view of one Hyper-Kamiokande water Cherenkov detector [1]. Our protocollaboration plans to construct two detector tanks consecutively, aiming the obsevations start at 2026 and 2032 respectively. Each tank will provide the fiducial volume of $0.187 \mathrm{Mt}$ ultra pure water, with the dimensions of the $74 \mathrm{~m}(\mathrm{D}) \times 60 \mathrm{~m}(\mathrm{H})$.

\section{Solar Neutrino}

The Sun is burning and emitting neutrinos with the nuclear fusion reactions, which are called as the pp-chain and the $\mathrm{CNO}$ cycle and can be summarized: $4 p \rightarrow \alpha+2 e^{+}+2 v_{e}$. These processes are described with the standard solar model (SSM) [7]. The SSM provides the good prediction of 
flux and energy spectrum of solar neutrinos. Since the solar neutrinos with $\mathrm{E}_{v}>6.5 \mathrm{MeV}$ will be detectable with Hyper-K, our dominant observation will be boron- 8 neutrinos. They are observed through neutrino-electron elastic scattering, $v+e \rightarrow v+e$. The energy, direction and time of the original neutrinos are measured through the recoil electron. About $70 v$-e scattering events will be observed in a day at each HK tank, while $15 v$ events/day are observed at SK-I to SK-IV.

One of the major motivations of solar neutrino study is the test of the solar model predictions. Because of its high penetration power, neutrino is the unique prove for the current activity of the solar core, where they are generated. Several precise measurements of solar neutrinos would be possible with Hyper-K and its high statistics, e.g. the undiscovered hep process neutrino generated in ${ }^{3} \mathrm{He}+p \rightarrow{ }^{4} \mathrm{He}+e^{+}+v_{e}$ reaction and the seasonal variation of the ${ }^{8} \mathrm{~B}$ neutrino flux. Another major motivation is the study of neutrino properties themselves. Super-K, SNO [8] and several experiments $[9,10,11]$ have been measured the neutrino oscillation on the solar neutrinos. A recent result of the oscillation analysis among solar neutrino experiments is following: $\sin \theta_{12}=0.311_{-0.014}^{+0.014}$, $\Delta m_{21}^{2}=4.85_{-0.59}^{+1.4} \times 10^{-5} \mathrm{eV}^{2}$ [12]. On the other hand, a reactor neutrino experiment, KamLAND, also measured these oscillation: $\tan ^{2} \theta_{12}=0.436_{-0.029}^{+0.029}, \Delta m_{21}^{2}=7.53_{-0.18}^{+0.18} \times 10^{-5} \mathrm{eV}^{2}$ [12]. Figure 2 on the next page shows the comparison of these two measurement results and these combined result. The $\sim 2 \sigma$ tension between these $\Delta m_{21}^{2}$ measurement could be tested by Hyper-K. The tension is derived from the asymmetry of the solar neutrino flux during day and night (day-night asymmetry), which ws indicated by Super-K [13]. The asymmetry would arises from the terrestrial matter effect.With Hyper-K, the day-night asymmetry effect can be measured precisely with the large statistics. Assuming the current solar best $\Delta m_{21}^{2}$ parameter, our measurement will be precise enough to separate itself from the current KamLAND best value about $4 \sigma$ with 10 years observation. The difference of $\mathrm{P}_{v_{e} \rightarrow v_{e}}$ in solar neutrino oscillation and $\mathrm{P}_{\bar{v}_{e} \rightarrow \bar{v}_{e}}$ in reactor neutrino will be a test of CPT violation at neutrinos. The solar neutrino energy spectrum upturn, where the beyond standard model physics could be, is also the interesting physics properties. The discovery sensitivity will be about $3 \sigma(4 \sigma)$ after the 10 years solar neutrino measurement with $4.5 \mathrm{MeV}(3.5 \mathrm{MeV})$ threshold.

\section{Supernova Neutrinos}

Core collapse supernova explosions are the last process in the evolution of massive stars $(>8$ $\mathrm{M}_{\odot}$ ). The energy released by a supernova is estimated to be $\sim 3 \times 10^{53} \mathrm{ergs}$ and $99 \%$ of the energy is carried out by all three types of neutrinos and anti-neutrinos. The detection of supernova neutrinos gives direct information of energy flow during the explosions. From SN1987a, the Kamiokande, IMB, and Baksan experiments observed 25 neutrino events. It proved the basic scenario of the supernova explosion was correct. However, close to three decades later the detailed mechanism of explosions is still not known. The observation of new supernova with the large neutrino detector is desired. The multi-messenger observation with visible light, gamma-ray, x-ray, gravitational wave and Hyper-K will also reveal the supernova explosion in details.

The first and direct observation of supernova neutrinos is about the supernova burst neutrinos, which are released in several seconds after its onset of a burst. About $90 \%$ of signals at Hyper-K is inverse beta reaction $\left(\bar{v}_{e}+p \rightarrow e^{+}+n\right)$. For each full volume of two inner detectors, we ex- 


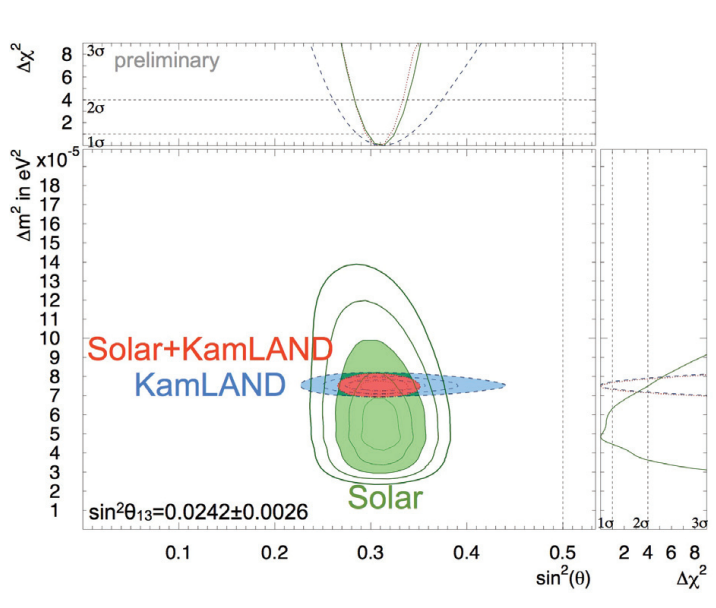

Figure 2: Neutrino oscillation parameter allowed region from all the solar experiments (green), KamLAND (blue) and Solar+KamLAND (red) from 1 to $5 \sigma$ lines and $3 \sigma$ area are shown[12].

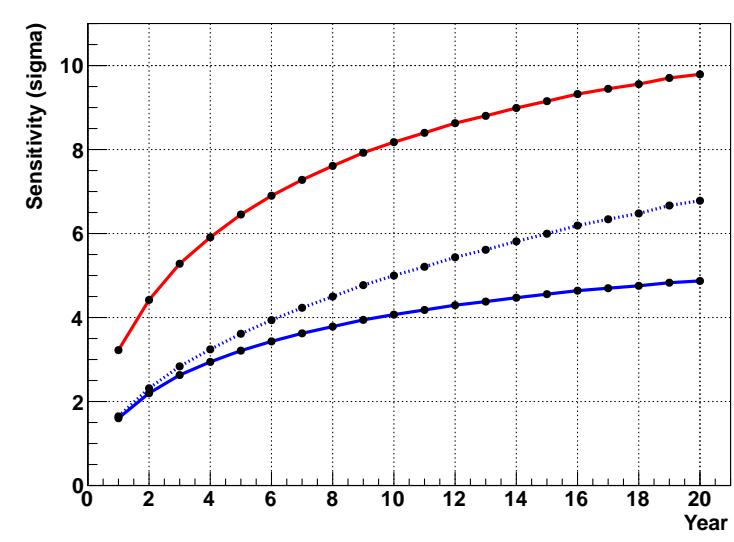

Figure 3: Day-night asymmetry observation sensitivity as a function of observation time. The red line shows the sensitivity from the no asymmetry, while the blue line shows from the asymmetry expected by the reactor neutrino oscillation. The solid line shows that the systematic uncertainty is $0.3 \%$, while the dotted line shows the $0.1 \%$ case.

pect to see about 49,000-68,000 inverse beta events, 2,100-2,500 $v$-e scattering events, $80-4,100$ $v_{e}+{ }^{16} \mathrm{O}$ CC events, and 650-3,900 $\bar{v}_{e}+{ }^{16} \mathrm{O}$ CC events, in total 52,000 79,000 events for a supernova explosion at halfway across our galaxy $(10 \mathrm{kpc})$. The statistical error will be small enought to compare several SN models, and so Hyper-K should give crucial data for further model predictions. A new characteristic modulation of the supernova neutrino flux also can be tested with HK, which the recent computer simulations predict. In past simulation, the deceleration of the shock wave and resulting failed explosion were annoying problems. Recent simulations suggest that the shock wave will be heated efficently by neutrinos to revival, due to the physical motions in a supernova, Standing Accretion Shock Instability (SASI) or convection, rotation os supernova are the examples. These models also predict the characteristic frequency modulation of neutrino flux, due to the motions in supernovae. The detection of these modulation will prove the neutrino as the driver of supernova explosions. Other topics for astrophysics and particle physics also can be examined, e.g. direct observation of black hole formations and mass hierarchy of neutrinos.

Another observation target is about the supernova relic neutrinos (SRN), produced by all past supernova explosions since the beginning of the universe and diffused. They must fill the universe and their flux is estimated to be a few tens $/ \mathrm{cm}^{2} / \mathrm{sec}$. SRN contains the information of its origins, i.e. the star formation rate, energy spectrum of supernova burst neutrinos, and the fraction of strange supernova explosions like dim supernovae or black hole formations. Although searches for SRN have been conducted at large underground detectors, no evidence of SRN signals has yet been obtained, because of the small flux of SRN. With incoming detector update, Gd-loaded SuperKamiokande (SK-Gd) can be the discoverer of SRN. The number of event in their detector will be 0.8-5 events/year above $10 \mathrm{MeV}$. Even though, it is still very interesting physics theme to measure and determine the precise flux of SRN. $\sim 70 \mathrm{SRN}$ events are expected at 16-30 MeV with 1 tank and 10 years observation. The significance will be $4.2 \sigma$ and enough for confirming the discovery. Further studies for astro and particle physics topics will be performed. 


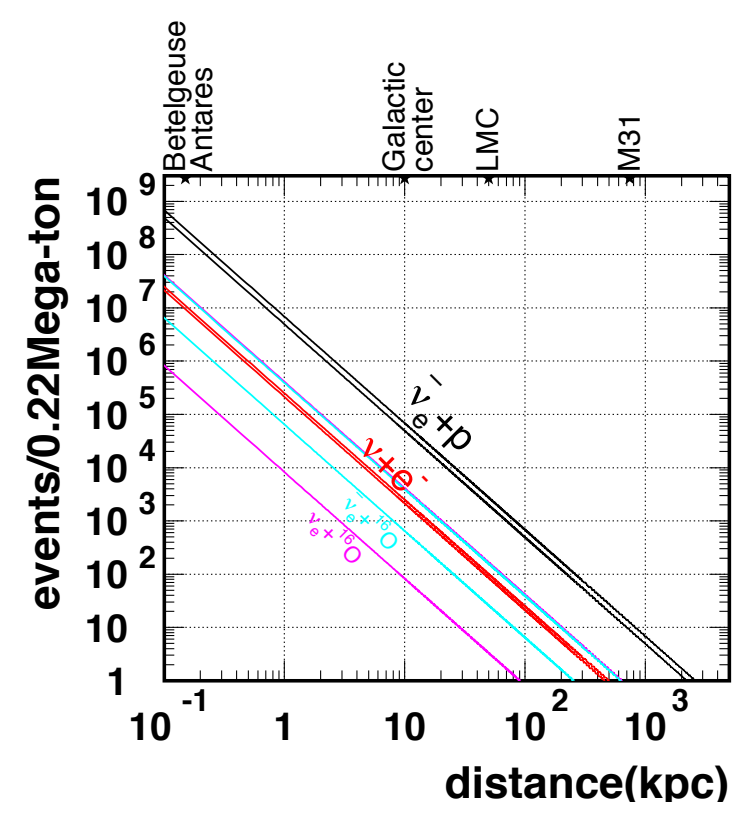

Figure 4: Expected number of supernova burst events for each interaction as a function of the distance to a supernova. The band of each line shows the possible variation due to the assumption of neutrino oscillations.

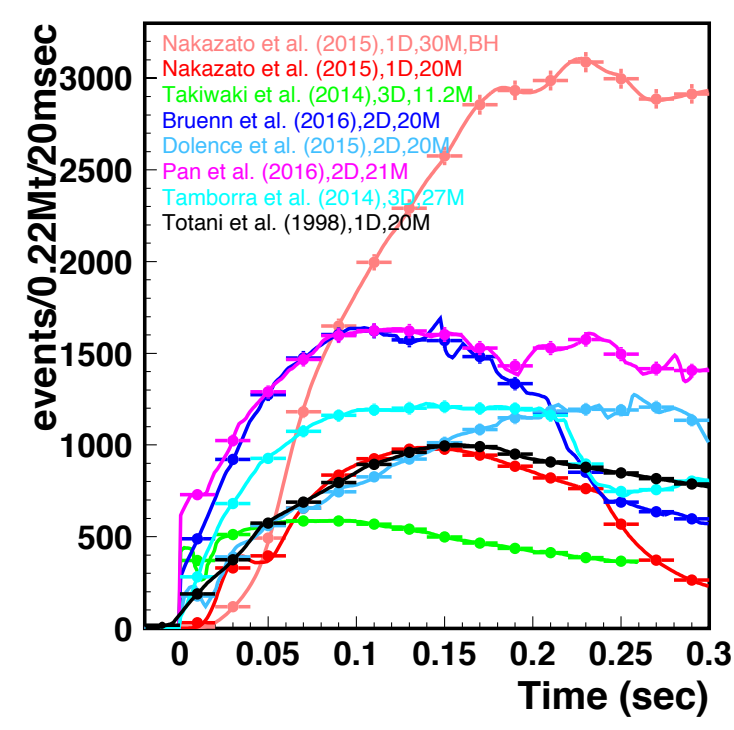

Figure 5: Inverse beta event rate predicted by supernova simulations for the first 0.3 seconds after the onset of a $10 \mathrm{kpc}$ distant burst. The error bars shows the statistic error.

\section{Summary}

Hyper-Kamiokande is a next generation large water Cherenkov detector. Several studies are being performed, e.g. photosensor R\&D, design and physics optimization. Astrophysical neutrino measurement is one of the features of Hyper-K. We will provide the unique information for solar, supernova burst and supernova relic neutrinos. Hyper-K will play remarkable role in the next neutrino physics frontier for both of particle physics and neutrino astrophysics.

\section{References}

[1] K. Abe et al., Letter of Intent: The Hyper-Kamiokande Experiment - Detector Design and Physics Potential - 1109.3262.

[2] Hyper-Kamiokande Working Group collaboration, K. Abe et al., A Long Baseline Neutrino Oscillation Experiment Using J-PARC Neutrino Beam and Hyper-Kamiokande, 2014, 1412.4673.

[3] HYPER-KAMIOKANDE collaboration, Hyper-Kamiokande Design Report, KEK-PREPRINT-2016-21, ICRR-REPORT-701-2016-1 (2016) .

[4] SUPER-KAMiOKANDE collaboration, Y. Fukuda et al., The Super-Kamiokande detector, Nucl. Instrum. Meth. A501 (2003) 418-462.

[5] Hyper-Kamiokande Proto collaboration, Y. Nishimura, New $50 \mathrm{~cm}$ Photo-Detectors for Hyper-Kamiokande, PoS ICHEP2016 (2017) 303.

[6] HyPER-KAmiokAnde PROTO collaboration, A. C. Ruggeri, R\&D studies of new photosensors for the Hyper-Kamiokande detector, Springer Proceedings in Physics TIPP2017 (2017) To be published. 


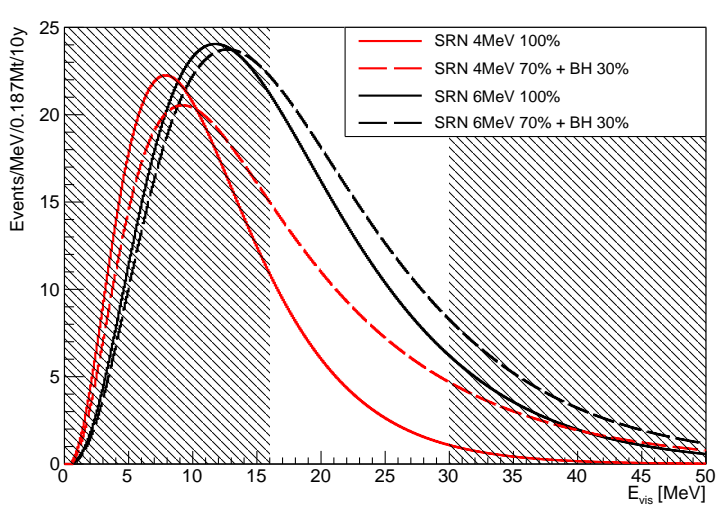

Figure 6: The expected SRN signal in 1 tank FV and 10 years observation. Black line shows the case of supernova neutrino temperature of $6 \mathrm{MeV}$, and red shows the case of $4 \mathrm{MeV}[14,15]$. Solid (dashed) line corresponds to the case, in which all the corecollapse supernovae emits neutrinos with the particular energy (30\% of them form black hole and emits higher energy neutrinos with the temperature of $8 \mathrm{MeV}$ ). Shaded energy region shows the range out of SRN search window at Hyper-K.

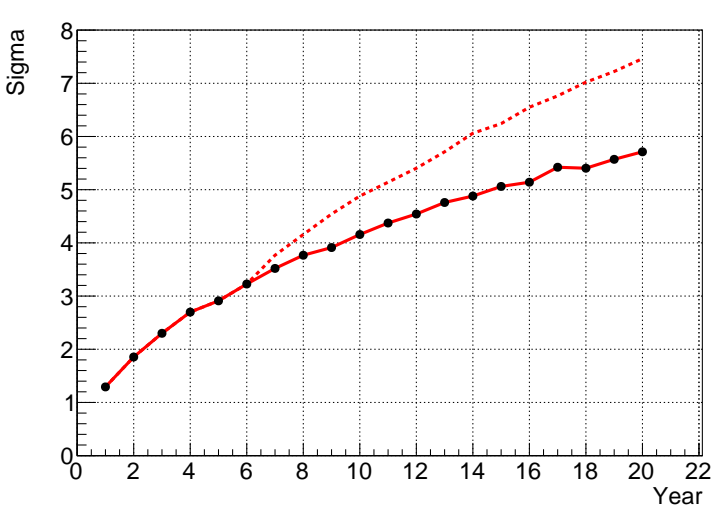

Figure 7: The figure shows the number of observed the SRN discovery sensitivity as a function of o bservation period. Red solid line shows the continuous measurement with 1 tank and red dashed line shows the scenario with 2 tanks after 6 years, respectively.

[7] J. N. Bahcall, A. M. Serenelli and S. Basu, 10,000 standard solar models: a Monte Carlo simulation, Astrophys. J. Suppl. 165 (2006) 400-431, [astro-ph/ 0511337 ].

[8] SNO collaboration, B. Aharmim et al., Combined Analysis of all Three Phases of Solar Neutrino Data from the Sudbury Neutrino Observatory, Phys. Rev. C88 (2013) 025501, [1109. 0763].

[9] B. T. Cleveland, T. Daily, R. Davis, Jr., J. R. Distel, K. Lande, C. K. Lee et al., Measurement of the solar electron neutrino flux with the Homestake chlorine detector, Astrophys. J. 496 (1998) 505-526.

[10] SAGE collaboration, J. N. Abdurashitov et al., Measurement of the solar neutrino capture rate with gallium metal. III: Results for the 2002-2007 data-taking period, Phys. Rev. C80 (2009) 015807, [0901.2200].

[11] G. Bellini et al., Precision measurement of the 7Be solar neutrino interaction rate in Borexino, Phys. Rev. Lett. 107 (2011) 141302, [1104.1816].

[12] Y. Koshio, Solar results from Super-Kamiokande, AIP Conf. Proc. 1666 (2015) 090001.

[13] Super-KAmioKande collaboration, A. Renshaw et al., First Indication of Terrestrial Matter Effects on Solar Neutrino Oscillation, Phys. Rev. Lett. 112 (2014) 091805, [1312 . 5176].

[14] S. Horiuchi, J. F. Beacom and E. Dwek, The Diffuse Supernova Neutrino Background is detectable in Super-Kamiokande, Phys. Rev. D79 (2009) 083013, [0812.3157].

[15] S. HoriuchiPrivate communication (2017) . 